\title{
Self-Assembly of Periodic Bicomponent Wires and Ribbons**
}

\author{
Marta E. Cañas-Ventura, Wende Xiao, Daniel Wasserfallen, Klaus Müllen, Harald Brune, \\ Johannes V. Barth, and Roman Fasel*
}

The success of modern supramolecular chemistry is largely based on the enhanced stability and enlarged number of distinct heteromolecular species generated from relatively weak, noncovalent interactions such as hydrogen bonding and metal-ligand interactions. ${ }^{[1]}$ In recent years, the transfer and adaptation of the corresponding concepts to the formation of two-dimensional (2D) supramolecular structures on surfaces has been intensively explored. ${ }^{[2]} \mathrm{A}$ number of studies on the adsorption of binary molecular mixtures on well-characterized single-crystal surfaces have been performed. ${ }^{[3]}$ In a few cases, the successful formation of regular heteromolecular species through hydrogen-bonding interactions has been reported. ${ }^{[4]}$ The controlled organization of surface-supported supramolecular nanosystems over large length scales, however, has not been achieved so far.

Ordered arrays of supramolecular wires are among the most desirable artificially structured nanosystems, because of their potential as elemental building blocks in future device applications. The growth of one-dimensional (1D) adsorbed supramolecular structures has been achieved, albeit with chain lengths restricted to the size of atomic terraces. ${ }^{[5]}$ Longrange ordered 1D supramolecular structures require a template surface where steps induce order instead of destroying it. The fabrication of a regular superlattice of specific 1D surface-supported supramolecular wires based upon hydrogen-bonding interactions thus relies on a rational choice of both 1) the complementary building blocks for $1 \mathrm{D}$ multitopic

[*] M. E. Cañas-Ventura, Dr. W. Xiao, Dr. R. Fasel nanotech@surfaces Laboratory

Empa, Swiss Federal Lab. for Materials Testing and Research Feuerwerkerstrasse 39, 3602 Thun (Switzerland)

Fax: $(+41)$ 33-228-6465

E-mail:roman.fasel@empa.ch

M. E. Cañas-Ventura, Prof. Dr. H. Brune

Institut de Physique des Nanostructures

Ecole Polytechnique Fédérale de Lausanne

Station 3, 1015 Lausanne (Switzerland)

Dr. D. Wasserfallen, Prof. Dr. K. Müllen

Max-Planck-Institut für Polymerforschung

Ackermannweg 10, 55128 Mainz (Germany)

Prof. Dr. J. V. Barth

Departments of Chemistry and Physics and Astronomy

University of British Columbia

Vancouver, BC V6T 1Z4 (Canada)

[***] We gratefully acknowledge the financial support from the European Commission (NMP3-CT-2004-001561 RADSAS). We thank P. Ruffieux, A. Arnau, and E. Ortega for stimulating discussions and for critically reading the manuscript.

$\square$ Supporting information for this article is available on the WWW under http://www.angewandte.org or from the author. hydrogen-bonding interactions, and 2) an appropriate template surface to guide the long-range growth of the supramolecular structures.

Herein we present ultrahigh vacuum (UHV) scanning tunneling microscopy (STM) investigations at $40 \mathrm{~K}$ (unless otherwise stated) of bimolecular wires and ribbons that form along the equidistant and parallel steps of an $\mathrm{Au}$ vicinal surface. Internal as well as long-range order of the binary wires and ribbons are reported. We use molecules exhibiting complementary end-group functionalities designed to form three hydrogen bonds. Deposition of both molecular species on $\mathrm{Au}(11,12,12)$ is shown to promote the anticipated formation of three hydrogen bonds per heteromolecular pair, in contrast to the two hydrogen bonds observed for singlecomponent deposits. Depending on coverage, the bicomponent system gives rise to a regular superlattice of $1 \mathrm{D}$ heteromolecular wires consisting of one or two molecular rows, as well as $2 \mathrm{D}$ supramolecular ribbons.

The chosen molecular species are 1,4-bis-(2,4-diamino1,3,5,-triazine)-benzene (BDATB; Figure 1a) and 3,4,9,10perylenetetracarboxylic diimide (PTCDI; Figure 1d). PTCDI exhibits a -CO-NH-CO- (imide) sequence on the two opposite sides with a $\mathrm{NH}$ hydrogen-bond donor (D) and two $\mathrm{CO}$ hydrogen-bond acceptors (A), thus giving rise to the well-known A-D-A sequence. ${ }^{[1,6]}$ BDATB was synthesized (see the Supporting Information) to provide interaction selectivity by means of complementary end-group functionalities (NH-N-NH) on the opposite sides of the molecules, which correspond to a D-A-D hydrogen-bonding sequence. The high suitability of the $\mathrm{Au}(11,12,12)$ surface as a template for the growth of long-range-ordered molecular structures is derived from its large extended double periodicity: 5.8-nmwide $\mathrm{Au}(111)$ terraces separated by monoatomic steps as well as periodic truncated "V"-shape discommensuration lines which indicate the border between face-centered cubic (fcc) and hexagonal close-packed (hcp) stacking domains (see the Supporting Information). ${ }^{[7]}$ The low reactivity of $\mathrm{Au}(111)$ is well suited for the formation of organic molecular nanostructures. ${ }^{[8]}$ Deposited molecules are generally highly mobile at room temperature, and at higher coverages form islands in which the order is predominantly determined by intermolecular interactions. Furthermore, reconstruction patterns and low-coordinated $\mathrm{Au}$ atoms at step edges represent preferential nucleation sites for the growth of supramolecular structures. ${ }^{[5,9]}$

In a first step, we investigated the homomolecular supramolecular structures resulting from the self-assembly of each molecular species deposited as a unique adsorbate on the $\mathrm{Au}(11,12,12)$ template surface (Figure 1). At coverages of approximately 0.5 monolayer (ML), BDATB and PTCDI 

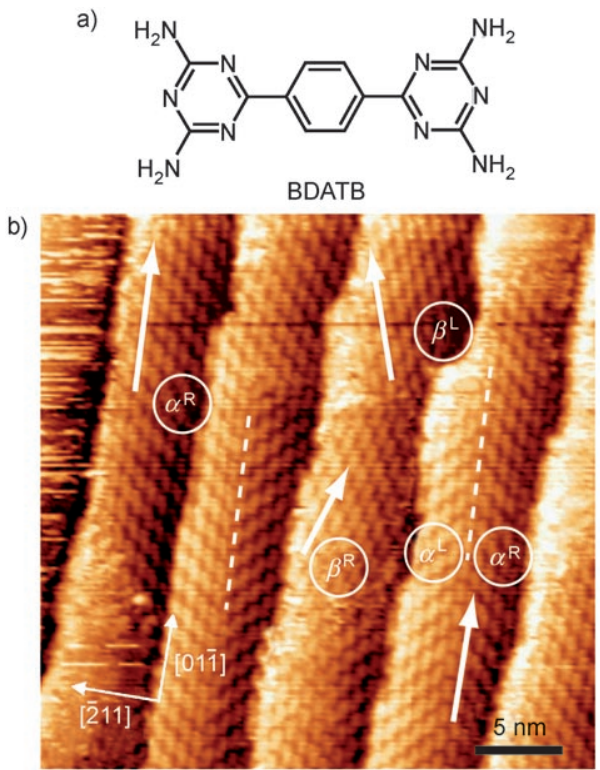

c)

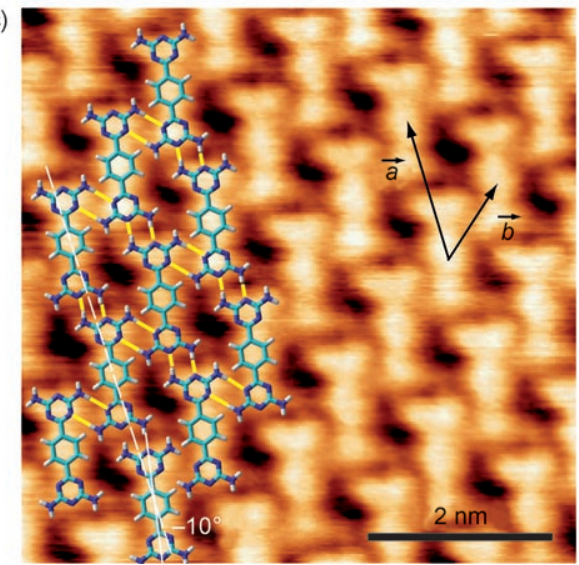

d)

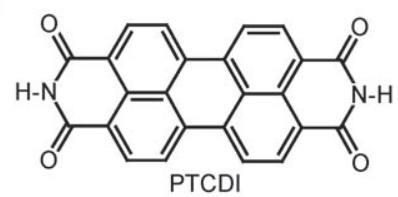

e)

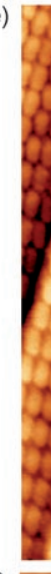

f)

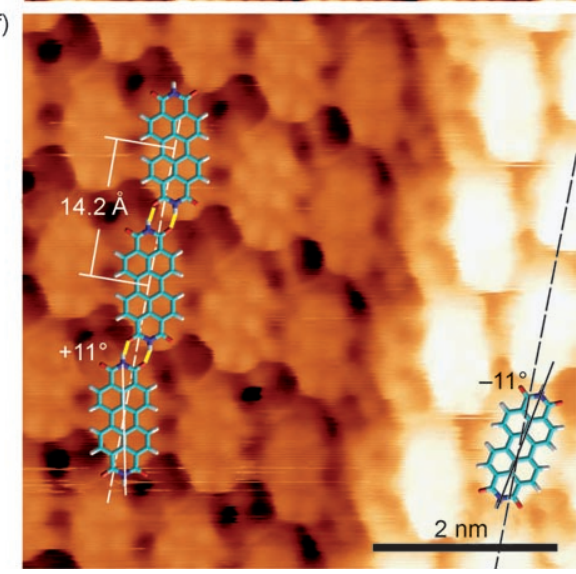

Figure 1. Single-component superlattice structures. a) Chemical structure of BDATB. b) STM image of $0.5 \mathrm{ML}$ BDATB adsorbed at RT on $\mathrm{Au}(11,12,12)$ showing several domains with different high-symmetry directions (white arrows) $\alpha$ and $\beta$. Mirror domain boundaries (dashed lines) and the high-symmetry directions of the underlying template surface are indicated (bias voltage $0.7 \mathrm{~V}$, tunneling current $0.1 \mathrm{nA}$, scanned at RT). c) High-resolution STM image. Unit-cell vectors: $\vec{a}$ and $\vec{b}$. A proposed model for the hydrogen bonds stabilizing the structure is superposed $(1.55 \mathrm{~V}, 0.025 \mathrm{nA})$. d) Chemical structure of PTCDI. e) STM image of a part of a PTCDI island covering several $A u(11,12,12)$ terraces $(-0.5 \mathrm{~V}, 0.04 \mathrm{nA})$. f) High-resolution STM image. The brighter molecular rows on the right-hand side lie on the upper terrace. Both canted configurations $\left( \pm 11^{\circ}\right)$ are defined with respect to the direction of the step edge $(0.06 \mathrm{~V}, 0.09 \mathrm{nA})$.

both self-assemble into extended domains. Two different orientational domains ( $\alpha$ and $\beta$ ) are identified for BDATB, with left $(\mathrm{L})$ and right $(\mathrm{R})$ staggering of the molecules along the high-symmetry direction. The long molecular axes of BDATB are canted by $\pm(10 \pm 2)^{\circ}$ with respect to the highsymmetry directions. By averaging the intermolecular distances determined from several STM images, the unit-cell vectors $\vec{a}$ and $\vec{b}$ are found to be $|\vec{a}|=(14.5 \pm 0.2) \AA$ and $|\vec{b}|=(10.1 \pm 0.2) \AA$, with an angle of $(50 \pm 2)^{\circ}$ in between. A twofold hydrogen-bonding pattern along the high-symmetry direction is suggested $(\mathrm{N}-\mathrm{H} \cdots \mathrm{N}$ distance of $(3.1 \pm 0.2) \AA)$ together with four additional hydrogen bonds across the rows $(\mathrm{N}-\mathrm{H} \cdots \mathrm{N}$ distance of $(3.3 \pm 0.2) \AA) \cdot{ }^{[10]}$ Deposition of BDATB results in a rearrangement of the step edges, which leads to a

deviation from the nominal terrace width in some regions. This effect might be related to the propensity of gold to act as an acceptor in N$\mathrm{H} \cdots \mathrm{Au}^{-}$hydrogen bonds. ${ }^{[11]}$

Deposition of PTCDI leads to domains oriented along the steps which can extend over several terraces (Figure 1e). As indicated in Figure $1 \mathrm{f}$, the long axis of each PTCDI molecule is canted by $\pm(11 \pm 2)^{\circ}$ with respect to the high-symmetry direction. Both orientations can alternate from row to row and seem to adapt such that there is always space for an integer number of PTCDI rows. The averaged intermolecular distance along the rows is $(14.2 \pm 0.2) \AA$. Pairs of $\mathrm{N}-\mathrm{H} \cdots \mathrm{O}$ hydrogen bonds (length of $(2.7 \pm 0.2) \AA)$ are formed at both ends of the PTCDI molecules. Relatively weak hydrogen-bonding interactions between adjacent PTCDI rows is suggested (C$\mathrm{H} \cdots \mathrm{O}$ distance of $(3.0 \pm 0.2) \AA)$. We believe that the molecular canting results from intermolecular forces rather than the substrate, since similar arrangements have been found for the same molecule adsorbed on other surfaces. ${ }^{[12]}$ The main difference with our system is given by the anisotropy of the surface which arises from the presence of a regular array of monoatomic steps. The close agreement between the PTCDI rows observed on this surface and the row motif observed in crystalline PTCDI further confirms the importance of intermolecular hydrogen-bonding interactions in surface ordering.

Figure 2 shows STM images of a highly ordered heteromolecular superlattice resulting from the deposition of PTCDI (50\%) and BDATB $(50 \%)$ slightly below $1 \mathrm{ML}$. The deposition sequence of the two components is unimportant for the formation of the mixed network - both molecules are mobile enough to intermix homogeneously whenever the stoichiometry is close enough to $1: 1$. Annealing further enhances the molecular mobility and increases the length scale on which the order of the mixed layer prevails; the optimum annealing temperature is $390 \mathrm{~K}$, for which an average of only one stacking fault per $10000 \mathrm{~nm}^{2}$ is found. The desorption temperature of approximately $490 \mathrm{~K}$ for BDATB (>520 K for PTCDI) defines the temperature stability limit of the system. In Figure 2, the brighter spots are the fingerprints of individual PTCDI molecules, which alternate with dimmer 


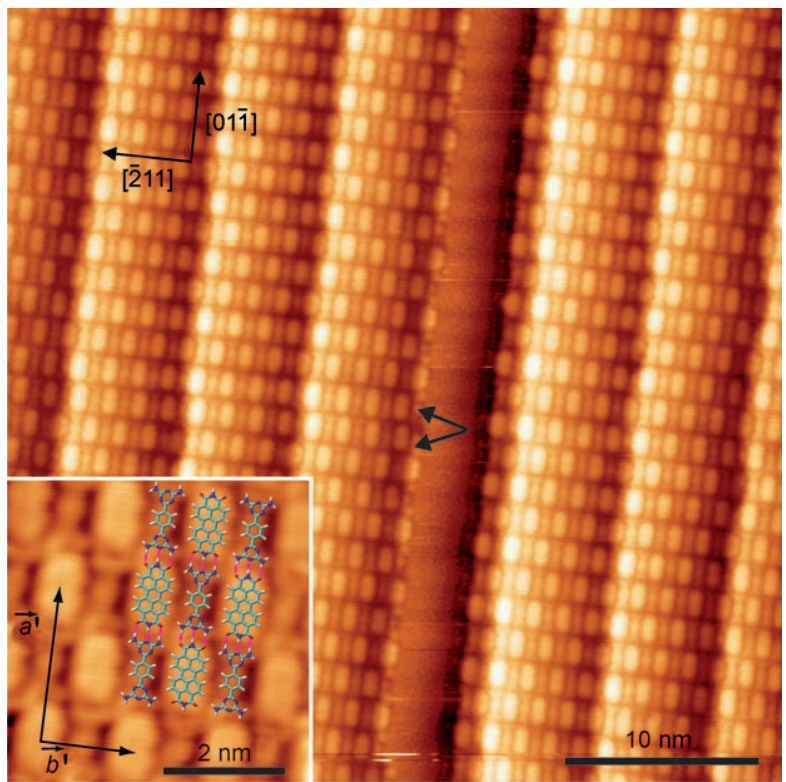

Figure 2. BDATB and PTCDI bicomponent supramolecular organization on $\mathrm{Au}(11,12,12)$ at $390 \mathrm{~K}$. STM image of seven terraces fully covered with the heteromolecular superlattice. One terrace (middle) shows only two molecular rows. The small arrows indicate a stacking fault propagating over several terraces $(-0.9 \mathrm{~V}, 0.034 \mathrm{nA})$. Inset: Highresolution STM image $(-0.4 \mathrm{~V}, 0.084 \mathrm{nA})$ and corresponding structural model of the binary supramolecular lattice with three hydrogen bonds between the heteromolecular pairs.

spots of the BDATB molecules. The alternating PTCDIBDATB sequence along both directions of the 2D ribbons can be described by a rectangular lattice, with (nonprimitive) unit-cell vectors $\vec{a}^{\prime}$ and $\vec{b}^{\prime}$ as shown in the inset. The lengths of these vectors are found to be $\left|\vec{a}^{\prime}\right|=(28.1 \pm 0.2) \AA$ and $\left|\vec{b}^{\prime}\right|=(15.2 \pm 0.1) \AA$, which correspond to hydrogen-bonding distances of $(2.8 \pm 0.2) \AA$ for the $\mathrm{N}-\mathrm{H} \cdots \mathrm{O}$ and $\mathrm{N} \cdots \mathrm{H}-\mathrm{N}$ bonds, which are similar to hydrogen-bond lengths reported in other studies. ${ }^{[1 \mathrm{c}, 13]}$ Only a single terrace near the middle of the image is not completely covered by molecules in Figure 2. A closer inspection reveals that it contains exactly two rows of alternating PTCDI and BDATB molecules. STM images show that terraces are either covered by one or two rows depending on the coverage, starting from the lower part of the step edges, or they are fully covered at higher coverages. Since both molecular species are geometrically symmetric and exhibit complementary end-group functionalities, a comparison with the single-component adsorption behavior leads us to attribute the directionality of the bicomponent system to the anticipated formation of the threefold hydrogen-bonding pattern. A tentative model is superimposed in the inset.

In addition to the threefold hydrogen-bonding pattern, the regular array of steps on the $\mathrm{Au}(11,12,12)$ template surface plays an important role in imprinting the unidirectionality of the bicomponent supramolecular structure, which is not observed on a $\mathrm{Au}(111)$ surface (see the Supporting Information). $\mathrm{Au}$ atoms at the lower step edges exhibit an enhanced negative charge density, which is thought to be responsible for their different (often enhanced) chemical reactivity. ${ }^{[14]}$ Furthermore, adsorption at the lower step edge also offers a greater level of coordination between molecules and substrate atoms. Interestingly, the lateral interaction between molecules (in the direction perpendicular to the step edges) is also rather strong, and even extends across the step edges: It can frequently be observed that a stacking fault (for example, two PTCDI molecules in a row) does not only propagate across the entire terrace, but propagates to many neighboring terraces across several step edges (see Figure 2 and the Supporting Information).

Deposition of small amounts (around 0.3 ML) of PTCDI and BDATB in a 1:1 stoichiometry results in the formation of the anticipated $1 \mathrm{D}$ bimolecular wires. Figure 3 a clearly shows alternating brighter and darker protrusions along the steps which correspond to PTCDI and BDATB, respectively. Electronic effects, such as the local density-of-states modulation at steps or between fcc and hcp stacking areas, can generate preferential adsorption sites. ${ }^{[5,9 c, e]}$ In the present

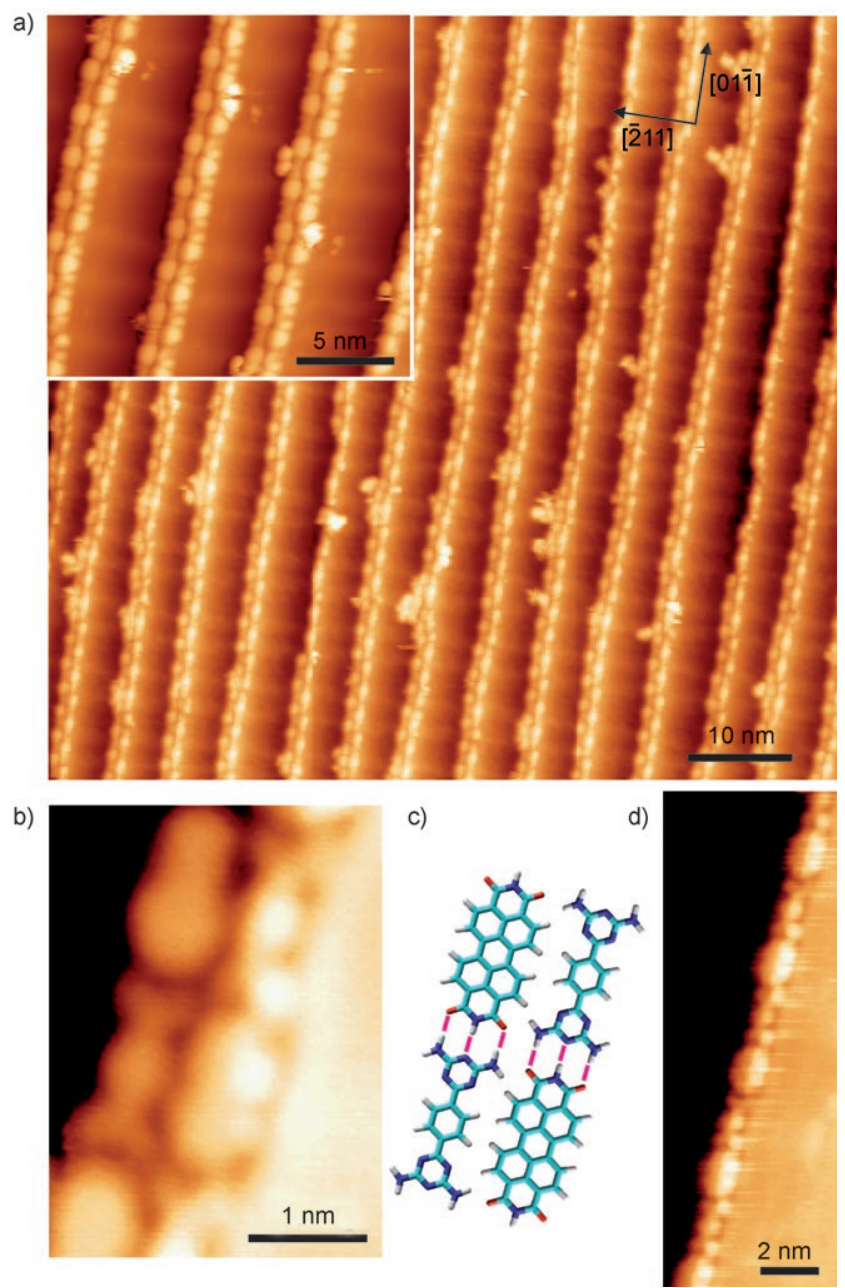

Figure 3. Double- and single-row heteromolecular wires running along the lower step edges of the $\mathrm{Au}(11,12,12)$ template surface. a) Overview STM image $(-1.7 \mathrm{~V}, 0.2 \mathrm{nA})$. Inset: STM image of a defect-free area. Brighter spots correspond to PTCDI molecules, darker ones to BDATB $(-1.1 \mathrm{~V}, 0.05 \mathrm{nA})$. b) Well-resolved individual molecules within the double-row wires $(-2.1 \mathrm{~V}, 0.13 \mathrm{nA})$. c) Model of the threefold hydrogen-bonding pattern suggested to promote the directionality of the structure. d) Close-up STM image of a well-ordered single-row wire exhibiting a perfect 1:1 stoichiometry, obtained after deposition of $0.15 \mathrm{ML}$ of PTCDI and BDATB (-0.9 V, $0.13 \mathrm{nA})$. 
heteromolecular system, however, intermolecular interactions appear to overrule the influence of the substrate reconstruction, since there is no apparent preference for either PTCDI or BDATB locating at fcc or hcp areas. This finding can be explained by the significant energy penalty of a gap in the hydrogen-bonding sequence $(>2 \times 0.37 \mathrm{eV}$, see the Supporting Information) compared to the small electronic potential energy difference (ca. $0.092 \mathrm{eV}^{[15]}$ ) between fcc and hcp regions. Figure $3 \mathrm{~b}$ highlights the intramolecular features already detectable on the larger scale images. The corresponding model in Figure $3 \mathrm{c}$ clarifies the structure that is stabilized by the targeted triple hydrogen-bonding pattern along the wire axis.

A further lowering of the surface coverage to $0.15 \mathrm{ML}$ leads to single-row bicomponent wires decorating the step edges (Figure $3 \mathrm{~d}$ ). In these single-row wires, the alternating order of PTCDI and BDATB along the wire axis is significantly lower than for the double-row wires; the average defect-free wire length is on the order of $10 \mathrm{~nm}$ for single-row wires and $30 \mathrm{~nm}$ for double-row wires (see the Supporting Information). Single-row wires are also significantly less straight than double-row wires because of the increased presence of homomolecular pairs, which give rise to the previously described canted structures. Therefore, we conclude that the presence of a second row stabilizes the ordering within the molecular wires, possibly as a consequence of some degree of cooperativity in the hydrogen-bonding interactions. ${ }^{[1,16]}$ This observation reinforces the idea that lateral adsorbate-adsorbate interactions play a non-negligible role. Nevertheless, there are still areas where the periodic order is preserved also for single-row bicomponent wires (Figure $3 \mathrm{~d}$ ).

To get a further understanding of the experimental observations, we performed molecular mechanics calculations at the AMBER level of theory for homo- and heteromolecular pairs (see the Supporting Information). ${ }^{[17]}$ The experimentally determined canted configurations are confirmed to be the most stable ones for homomolecular pairs, and the calculations rationalize the observed preference for heteroversus homomolecular formation which underlies the formation of the bicomponent wire: The three hydrogen bonds between PTCDI and BDATB are significantly stronger than the two hydrogen bonds between homomolecular BDATB and PTCDI pairs.

Strong and highly directional hydrogen-bonding networks are not only of fundamental importance in nature, but their efficiency in assisting electron-transfer processes makes them increasingly appealing for technological application inspired by biomimetic principles. ${ }^{[18]}$ We have demonstrated that the formation of specific 1D hydrogen-bonding networks and their long-range growth can be controlled by a rational design of complementary building blocks for $1 \mathrm{D}$ multitopic hydrogen bonding and the choice of an appropriate template surface. The resulting superlattice of single- and double-row heteromolecular wires exhibits an almost constant wire-towire separation over extended surface areas.

Received: October 4, 2006

Revised: December 8, 2006

Published online: February 2, 2007
Keywords: adsorption - hydrogen bonds - nanostructures · scanning probe microscopy · self-assembly

[1] a) J.-M. Lehn, Supramolecular Chemistry, Concepts and Perspectives, VCH, Weinheim, 1995; b) D. N. Reinhoudt, M. CregoCalama, Science 2002, 295, 2403; c) T. Steiner, Angew. Chem. 2002, 114, 50; Angew. Chem. Int. Ed. 2002, 41, 48; d) M. W. Hosseini, Acc. Chem. Res. 2005, 38, 313; e) P. Gamez, J. Reedijk, Eur. J. Inorg. Chem. 2006, 29; f) L. J. Prins, D. N. Reinhoudt, P. Timmerman, Angew. Chem. 2001, 113, 2446; Angew. Chem. Int. Ed. 2001, 40, 2382.

[2] For a recent review, see J. V. Barth, G. Costantini, K. Kern, Nature 2005, 437, 671.

[3] a) M. de Wild, S. Berner, H. Suzuki, H. Yanagi, D. Schlettwein, S. Ivan, A. Baratoff, H.-J. Guentherodt, T. A. Jung, ChemPhysChem 2002, 3, 881; b) D. Bonifazi, H. Spillmann, A. Kiebele, M. de Wild, P. Seiler, F. Cheng, H.-J. Gúntherodt, T. Jung, F. Diederich, Angew. Chem. 2004, 116, 4863; Angew. Chem. Int. Ed. 2004, 43, 4759; c) S. De Feyter, A. Miura, S. Yao, Z. Chen, F. Würthner, P. Jonkheijm, A. P. H. J. Schenning, E. W. Meijer, F. C. De Schryver, Nano Lett. 2005, 5, 77; d) J. C. Swarbrick, B. L. Rogers, N. R. Champness, P. H. Beton, J. Phys. Chem. B 2006, 110, 6110; e) J. Ma, B. L. Rogers, M. J. Humphry, D. J. Ring, G. Goretzki, N. R. Champness, P. H. Beton, J. Phys. Chem. B 2006, 110, 12207.

[4] a) J. A. Theobald, N. S. Oxtoby, M. A. Phillips, N. R. Champness, P. H. Beton, Nature 2003, 424, 1029; b) C. Bobisch, Th. Wagner, A. Bannani, R. Möller, J. Chem. Phys. 2003, 119, 9804; c) H. Ohtani, R. J. Wilson, S. Chiang, C. M. Mate, Phys. Rev. Lett. 1988, 60, 2398; d) B. Xu, Ch. Tao, W. G. Cullen, J. E. ReuttRobey, E. D. Williams, Nano Lett. 2005, 5, 2207; e) S. Xu, M. Dong, E. Rauls, R. Otero, T. R. Linderoth, F. Besenbacher, Nano Lett. 2006, 6, 1434.

[5] a) T. Yokoyama, S. Yokoyama, T. Kamikado, Y. Okuno, S. Mashiko, Nature 2001, 413, 619; b) R. Otero et al., Angew. Chem. 2004, 116, 2144; Angew. Chem. Int. Ed. 2004, 43, 2095; c) J. V. Barth, J. Weckesser, C. Cai, P. Günter, L. Bürgi, O. Jeandupeux, K. Kern, Angew. Chem. 2000, 112, 1285; Angew. Chem. Int. Ed. 2000, 39, 1230; d) J. V. Barth, J. Weckesser, G. Trimarchi, M. Vladimirova, A. De Vita, C. Cai, H. Brune, P. Günter, K. Kern, J. Am. Chem. Soc. 2002, 124, 7991; e) Q. Chen, N. V. Richardson, Nat. Mater. 2003, 2, 324; f) T. Kawai, H. Tanaka, T. Nakagawa, Surf. Sci. 1997, 386, 124; g) M. Böhringer, K. Morgenstern, W.-D. Schneider, R. Berndt, F. Mauri, A. De Vita, R. Car, Phys. Rev. Lett. 1999, 83, 324; h) D. L. Keeling, N. S. Oxtoby, C. Wilson, M. J. Humphry, N. R. Champness, P. H. Beton, Nano Lett. 2003, 3, 9.

[6] a) G. M. Whitesides, E. E. Simanek, J. P. Mathias, C. T. Seto, D. N. Chin, M. Mammen, D. M. Gordon, Acc. Chem. Res. 1995, 28, 37; b) G. M. Whitesides, J. P. Mathias, C. T. Seto, Science 1991, 254, 1312; c) D. C. Sherrington, K. A. Taskinen, Chem. Soc. Rev. 2001, 30, 83.

[7] S. Rousset, V. Repain, G. Baudot, Y. Garreau, J. Lecoeur, J. Phys. Condens. Matter 2003, 15, S3363.

[8] a) S. Clair, S. Pons, A. P. Seitsonen, H. Brune, K. Kern, J. V. Barth, J. Phys. Chem. B 2004, 108, 14585; b) M. Eremtchenko, D. Bauer, J. A. Schaefer, F. S. Tautz, New J. Phys. 2004, 6, 4.

[9] a) X. Chen, E. R. Frank, R. J. Hamers, J. Vac. Sci. Technol. B 1996, 14, 1136; b) J. I. Pascual, J. J. Jackiw, K. F. Kelly, H. Conrad, H.-P. Rust, P. S. Weiss, Phys. Rev. B 2000, 62, 12632; c) M. Vladimirova, M. Stengel, A. De Vita, A. Baldereschi, M. Böhringer, K. Morgenstern, R. Berndt, W.-D. Schneider, Europhys. Lett. 2001, 56, 254; d) A. Tamai, W. Auwärter, C. Cepek, F. Baumberger, T. Gerber, J. Osterwalder, Surf. Sci. 2004, 566-568, 633; e) W. Xiao, P. Ruffieux, K. Ait-Mansour, O. Gröning, K. Palotas, W. A. Hofer, P. Gröning, R. Fasel, J. Phys. Chem. B 
2006, 110, 21394; f) T. Yokoyama, T. Kamikado, S. Yokoyama, S Mashiko, J. Chem. Phys. 2004, 121, 11993; g) N. Néel, J. Kröger, R. Berndt, Adv. Mater. 2006, 18, 174.

[10] a) L. M. A. Perdigão, E. W. Perkins, J. Ma, P. A. Stainec, B. L. Rogers, N. R. Champness, P. H. Beton, J. Phys. Chem. B 2006, 110, 12539; b) P. Jonkheijm, A. Miura, M. Zdanowska, F. J. M. Hoeben, S. De Feyter, A. P. H. J. Schenning, F. C. De Schryver, E. W. Meijer, Angew. Chem. 2004, 116, 76; Angew. Chem. Int. Ed. 2004, 43, 74; c) A. Cousson, B. Nicolaï, F. Fillaux, Acta Crystallogr. Sect. E 2005, 61, 222.

[11] H. Nuss, M. Jansen, Angew. Chem. 2006, 118, 4476; Angew. Chem. Int. Ed. 2006, 45, 4369.

[12] a) C. Ludwig, B. Gompf, J. Petersen, R. Stohmaier, W. Eisenmenger, Z. Phys. B 1994, 93, 365; b) B. Uder, C. Ludwing, J. Petersen, B. Gompf, W. Eisenmenger, Z. Phys. B 1995, 97, 389; c) J. C. Swarbrick, J. Ma, J. A. Theobald, N. S. Oxtoby, J. N.
O'Shea, N. R. Champness, P. H. Beton, J. Phys. Chem. B 2005, 109, 12167.

[13] A. Ranganathan, V. R. Pedireddi, C. N. R. Rao, J. Am. Chem. Soc. 1999, 121, 1752.

[14] R. Smoluchowski, Phys. Rev. 1941, 60, 661.

[15] C. Didiot, Y. Fagot-Revurat, S. Pons, B. Kierren, C. Chatelain, D. Malterre, Phys. Rev. B 2006, 74, 081404(R).

[16] a) R. Otero, M. Schöck, L. M. Molina, E. Laegsgaard, I. Stensgaard, B. Hammer, F. Besenbacher, Angew. Chem. 2005, 117, 2446; Angew. Chem. Int. Ed. 2005, 44, 2270; b) Y.-F. Chen, J. J. Dannenberg, J. Am. Chem. Soc. 2006, 128, 8100.

[17] a) W. D. Cornell et al., J. Am. Chem. Soc. 1995, 117, 5179; b) HyperChem Release 7.5, Hypercube Inc., $1115 \mathrm{NW} 4$ th Street, Gainesville, Florida 32601 (http://www.hyper.com/).

[18] L. Sánchez, M. Sierra, N. Martín, A. J. Myles, T. J. Dale, J. Rebek, Jr., W. Seitz, D. M. Guldi, Angew. Chem. 2006, 118, 4753; Angew. Chem. Int. Ed. 2006, 45, 4637. 